\title{
University of Texas MD Anderson Cancer Center
}

National Cancer Institute

\section{Source}

National Cancer Institute. University of Texas MD Anderson Cancer Center. NCI

Thesaurus. Code C39557.

The mission areas of the University of Texas MD Anderson Cancer Center include patient care, research, education, and prevention, with an emphasis on cancer control and prevention trials. It strives to rapidly translate scientific knowledge into better cancer therapies and prevention strategies. It was named an $\mathrm{NCl}$-designated comprehensive cancer center in 1971. 